\title{
Recurrent Gliomas- A Short Review of Literature
}

\author{
Sudeb Mukherjee* \\ Department of Medicine, IPGMER and SSKM Hospital, India
}

Submission: June 22, 2018; Published: July 06, 2018

*Corresponding author: Sudeb Mukherjee, MBBS, MD(Medicine), DM-Post Doctorate Fellow-ICVS, IPGMER and SSKM Hospital, Kolkata-700020, India, Email: drsumukherjee@gmail.ciom

\begin{abstract}
Gliomas one of the commonest tumour account for $40-60 \%$ of all primary neoplasm of the central nervous system. Very little is known about pathological changes associated with recurrence of gliomas. Histological changes regarding this transformation also is not well documented Effect of radiotherapy and chemotherapy which results in decrease in cellularity, fibrosis, thickening of vessels are also not well documented. Paired study combining both primary and recurrent gliomas are sparse. Several genetic alterations have been mentioned in the causation and pathophysiology of recurrent gliomas. Starting from overexpression to mutation of p53 to Epidermal Growth Factor (EGFR), platelet derived growth factor- $\alpha$ (PDGF-A), PTEN, MDM2 all have mentioned several studies. This review article describes every aspect of recurrent gliomas in short span.
\end{abstract}

Keywords: Abdominal pain; Ovary; Torsion; Salpingitis; Schistosomiasis; Missionary Hospital; Operation; Uneventful recovery; Nigeria

\section{Introduction}

Gliomas account for $40-60 \%$ of all primary neoplasms of the central nervous system. It is the commonest human brain tumour is histologically divided into four grades (grade I to IV) according to W.H.O. classification [1], depending on criteria such as nuclear atypia, mitotic activity, necrosis and microvascular proliferation. Grade I include juvenile pilocytic astrocytoma (JPA) and subependymal giant cell astrocytoma (SEGA). Grade II includes diffuse fibrillary astrocytoma, Grade III anaplastic astrocytoma, Grade IV glioblastoma multiforme now known as glioblastoma. This classification also includes oligodendroglioma (grade II), anaplastic oligodendroglioma(grade-III), mixed oligoastrocytoma (grade-II), anaplastic oligoastrocytoma (grade-III), ependymoma (grade II), anaplastic ependymoma (grade III), myxopapillary ependymoma (grade I) and subependymoma (grade I).

Recurrences are fairly common in gliomas. Juvenile pilocytic astrocytoma (grade-I), astrocytoma (grade-II), oligodendroglioma (grade-II), myxopapillary ependymoma (grade-I) all have propensity to undergo recurrence. Sometimes they transform into high grade variety. These recurrent changes from their low-grade counterpart to high grade tumour (anaplastic variety or glioblastoma) adversely affect their prognosis.

Well differentiated diffuse astrocytoma may remain static or progress only slowly over a number of years. The mean survival is more than 5 years. Whereas survival in patient with anaplastic variety ranges from 2 to $5 y$ rs. Glioblatoma has a worse prognosis with a median survival of less than 1yr. But survival may be prolonged to 15 months with recent advancement of treatment [2].

Oligodendrogliomas have a more benign course. For grade II oligodendroglioma, median survival is 7-8yrs and there are a substantial number of patients with prolonged survival $(>10$ yrs). For grade III or anaplastic variety median survival is approximately 3.9yrs [3].

For intracranial ependymoma in children $5 y$ r progression free survival is $50 \%$. Children affected during the first $2 y r s$ of life carry a particularly dismal prognosis. In adult patient's survival of 5 and $10 y$ rs is $57.1 \%$ and $45 \%$ respectively [4-6].

\section{Genetic alteration}

Certain genetic alterations correlate with the progression of infiltrating astrocytoma from low to high grade, which is part of the natural course of the disease in many patients. Among the alterations that are most common in the low grade astrocytomas are mutations affecting p53 and overexpression of platelet derived growth factor- $\alpha$ (PDGF-A). Glioblastoma (WHO grade IV), the most frequent brain tumour in adult, accounting for approximately $12-15 \%$ of all intracranial neoplasms and $50-60 \%$ of all astrocytic tumour [7], is the least differentiated and most malignant neoplasm of astrocytic origin which typically affects adults and is preferentially located in the cerebral hemisphere. It is of two types one which arise de novo, called primary type (60\%), characterised by rapid onset usually less than 3 months 
and seen in older age (mean age 56yrs). Whereas secondary type (40\%) results of progression from a low-grade astrocytoma after 1 to $10 y$ rs (mean 4 to $5 y r s$ ), occur in younger adult $(<45 \mathrm{yrs})$. From prognostic point of view age of onset is the most important one, so recurrent or secondary glioblastoma has a better prognosis in comparison to primary one which is associated with EGFR overexpression and amplification [8]. While primary and secondary glioblastoma show some molecular distinctions, the molecular lesions found in the two types of glioblastoma tend to impinge on the same pathways. For example, whereas secondary glioblastomas usually have p53 mutations, primary type more commonly have amplifications of MDM2, a gene that encodes an inhibitor of p53. Similarly, while secondary glioblastomas have increased signalling through PDGF-A receptor, primary often have amplified or mutated epidermal growth factor receptor (EGFR) genes, which encodes aberrant forms of EGFR known as EGFRvIII. Both types of mutations lead to increased receptor tyrosine kinase activity and the activation of RAS and PI-3 kinase pathways, which stimulate the growth and survival of tumour cells. Based on whole genome sequencing, it is estimated that combinations of mutations that activate RAS and PI-3 kinase and inactivate p53 and RB are present in $80 \%$ to $90 \%$ of primary glioblastomas [9].

\section{Location}

Glioblastoma occurs most commonly in the subcortical white matter of the cerebral hemispheres, most frequently affected sites are temporal (31\%), parietal (24\%), frontal (23\%) and occipital (16\%) lobe. Combined fronto-temporal location is particularly typical [10]. Tumour infiltration often extends into the adjacent cortex, the basal ganglia \& contralateral hemisphere. Intraventricular glioblastomas are exceptional. Glioblastoma rapidly invade the neighbouring brain structure particularly contralateral hemisphere creating a symmetrical bilateral lesion (butterfly glioma). Despite its rapid infiltrative growth, the glioblastoma tends not to invade the subarachonoid space and consequently, rarely metastasise via CSF. Extension within \& along the perivacular spaces is another typical mode of infiltration but, invasion of vessel wall does not seem to occur and haematogenous spread to extraneural tissue is very rare in patients without previous surgical intervention [11].

\section{Radiological features}

On CT scan glioblastoma typically present as irregular shaped lesion with a peripheral ring like zone of contrast enhancement around a dark central area of necrosis that is usually hypodense. On T1 weighted MRI images, the contrast enhanced structure to the cellular and highly vascularised peripheral area of neoplasm. A study of untreated neoplasms on whole brain sections clearly shows that this ring structure does not represent the outer tumour border, since infiltrating glioma cells can easily be identified within and occasionally beyond a $2 \mathrm{~cm}$ margin [12]. In $\mathrm{T}_{2}$ weighted images this zone is broader, less well defined, and overlaps with surrounding vesogenic oedema. On PET scan regional glucose consumption closely correlates with cellularity and reduced survival [13].

\section{Histopathology}

On gross glioblastoma are poorly delineated, the cut surface shows a variable colouration with peripheral greyish tumour masses, yellow necrosis and single or multiple haemorrhages. The central necrosis may occupy as much as $90 \%$ of the total tumour mass. Macroscopic cysts, if present contain a turbid fluid and liquefied necrotic tumour tissue, quite in contrast to the well delineated retention cysts in low grade astrocytomas. Haemorrhages are usually small and dispersed throughout the neoplasm. However extensive haemorrhages may occur and evoke stroke-like symptoms which are sometimes the first clinical sign of tumour metastasis. On histological ground it is characterised by hypercellularity, nuclear pleomormphism, brisk mitotic activity, prominent microvascular proliferation and necrosis [14].

\section{Loss of heterozygosity ( $\mathrm{LOH})$}

$\mathrm{LOH}$ on chromosome arm $10 \mathrm{q}$ is the most frequent gene alteration for both primary and secondary glioblastomas; it occurs in $60-90 \%$ of cases. This mutation appears to be specific for glioblastoma multiforme and is found rarely in other tumor grades. This mutation is associated with poor survival. LOH at $10 \mathrm{q}$ plus 1 or 2 of the additional gene mutations appear to be frequent alterations and are most likely major players in the development of glioblastomas.

\section{p53}

p53 gene is located in chromosome 17. This tumour suppressor gene is responsible for cell cycle arrest, apoptosis, neovascularisation and also acts as a positive or negative regulator of transcription of other genes. p53 function in cancers can be lost by various mechanisms, including lesions that prevent activation of $p 53$, mutations within the $p 53$ gene (which encodes p53) itself or mutations of downstream mediators of p53 function. Analysis of many tumours has shown that $p 53$ is mutated in about half of all cancers, resulting in loss of apoptotic function. From the data available, it would seem that only 5\% of p53 mutations are found in the regulatory domains (amino terminus, amino acids 1-99; carboxyl terminus, amino acids 301393 ), whereas $95 \%$ of the mutations occur in the central region of $p 53$, which is responsible for sequence-specific DNA binding (amino acids 100-300). However, much of this information was derived from sequence analysis that included only exons 5-8 within the $p 53$ gene, and examination of the whole $p 53$ coding sequence is beginning to reveal an increasing number of p53 mutations in the amino and carboxyl termini of the protein. It is possible that the true mutation incidence for $p 53$ in cancers is actually significantly higher than the current estimate of $\sim 50 \%$ indeed, recent studies that highlight the importance of mutations outside the central core [15]. Tumour associated mutations in 
p53 are predominantly point mutations (93.6\%) that result in single amino-acid substitutions - a mutational spectrum that is quite different from that seen in other tumour suppressor genes, in which large deletions or frameshift mutations tend to result in the complete loss of protein expression. Furthermore, certain p53 codons shown.

Unexpectedly high mutation frequency, with $28 \%$ of the mutations affecting only six residues - 175, 245, 248, 249, 273 and 282 - of p53. The result of the mutational inactivation of p53 by single-amino-acid substitutions is that many tumour cells retain the ability to express the mutant $\mathrm{p} 53$ protein. These proteins are often more stable than wild-type p53 and are present at very high levels in the tumour cell. One explanation for the selection of such mutations is that the mutant p53 proteins can act as dominant- negative inhibitors of wild-type p53, which functions as a tetramer. The observation that many tumours that harbour p53 point mutations also show loss of heterozygosity - effectively eliminating the wild-type allele indicates that the efficiency of dominant-negative inhibition might not be complete, and almost certainly depends on the nature of the initial point mutation. However, partial inactivation of wild-type p53 function by mutant p53 might allow for some selective advantage during tumour progression, and individual who are heterozygous for a dominant-negative $\mathrm{p} 53$ point mutant developed tumours without loss of the wild-type p53 allele [16].

Mutations in p53 were among the first genetic alterations identified in astrocytic brain tumors. The p53 gene appears to be deleted or altered in approximately $25-40 \%$ of all glioblastoma multiformes, more commonly in secondary glioblastoma multiformes. The p53 immunoreactivity also appears to be associated with tumors that arise in younger patients [17]. Alterations of p53 have been observed in human astrocytomas of each histological grade, suggesting that this event may occur early in the genesis of these tumors. Mutation of p53 gene, which is one of the most frequent genetic alterations in human malignancies, including gliomas, is often associated with overexpression of the p53 protein.

Study comparing the number of p53 protein immunopositive cells and histological grade of astocytomas has been reported, showing that mutations of p53 gene were independent of tumoar grade and survival. On the other hand, overexpression of p53 protein occurs without detectable TP53 mutation in astrocytomas. In cotrast to astrocytoma oligodendroglioma and ependymomas are very rarely associated with p53 aterations.

\section{Epidermal growth factor receptor (EGFR) gene}

The human epidermal growth factor receptor gene product (EGFR), a member of the ErbB family of receptor tyrosine kinases, is an integral component of signaling in epithelial cell proliferation. Stimulation of the receptor with EGF or other cognate ligands induces receptor dimerization and autophosphorylation, providing docking sites for SH2- containing adaptor proteins that mediate the activation of intracellular signaling pathway [18].

Signaling by EGFR and related receptors is initiated by ligand binding which causes the receptor to change its conformation into the active form that readily dimerizes. In the active dimer, the C-terminal parts of the extracellular moiety of the molecule are quite close, forcing the membrane spanning segments to be in close contact. In this way the intracellular parts, the tyrosine kinases, are held in such proximity that they can easily transphosphorylate and thus activate each other. The signaling power of these receptors therefore rests on the close proximity of the C-terminal ends of the extracellular parts of the dimer. With the help of the data from the crystal structure of the human EGFR, it is predicted that the structural environment of the amino acids Arg336 and Ser555 that are responsible for the oncogenic capacity of the EGFR family protein. The results of various study indicate that both mutations cause the destruction of at least one intramolecular disulfide bridge and thereby allow the generation of intermolecular disulfide bridges that lead to permanent dimmers formation and autophosphorylation [19].

Multiple genetic mutations are apparent, including both overexpression of the receptor as well as rearrangements that resulted in truncated isoforms. However, all the clinically relevant mutations appear to contain the same phenotype leading to increased activity. These tumors typically show a simultaneous loss of chromosome 10 but rarely a concurrent p53 mutation. Overexpression or activation mutations in this gene are more common in primary glioblastoma, with mutations appearing in $40-50 \%$ of these tumors. One such common variant, EGFRvIII, has shown promise as a target for tyrosine kinase inhibitors, immunotoxins, and peptide vaccines [20]. The most frequent genetic alteration associated with GBM is amplification of the epidermal growth factor receptor (EGFR) gene, which results in over expression of the EGFR. The majority of GBMs with EGFR amplification also contain the mutant EGFR gene, EGFRvIII, which is characterized by the deletion of exons 2-7, resulting in a frameshift deletion variant that has a truncated extracellular domain with ligand-independent constitutive activity. Previous work has shown that EGFR amplification is evident in all GBMs expressing EGFRvIII and GBMs lacking the amplified EGFR are not positive for EGFRvIII protein. The role of the over expressed EGFR (wild type) and the variant (vIII) receptor in malignant progression of glial tumors and their respective impacts on overall survival have been debated in the literature. Over expression of wild-type EGFR was not found to be an independent prognostic indicator of survival in several studies and some study were inconclusive. Some studies identified EGFR as a negative prognostic indicator of survival. In some of these studies, analysis was limited by small sample size, uncharacterized extent of surgical resection, and variable postoperative treatment. The prognostic impact of EGFRvIII has not been as extensively studied. 


\section{MDM2}

Amplification or overexpression of MDM2 constitutes an alternative mechanism to escape from p53 -regulated control of cell growth by binding to p53 and blunting its activity. Overexpression of MDM2 is the second most common gene mutation in glioblastoma multiformes and is observed in 10$15 \%$ of patients. Some studies show that this mutation has been associated with a poor prognosis [21].

\section{Platelet-derived growth factor-alpha (PDGF-alpha) gene}

The PDGF gene acts as a major mitogen for glial cells by binding to the PDGF receptor (PDGFR). Amplification or overexpression of PDGFR is typical (60\%) in the pathway leading to secondary glioblastomas.

\section{PTEN}

PTEN (also known as MMAC and TEP1) encodes a tyrosine phosphatase located at band 10q23.3. The function of PTEN as a cellular phosphatase, turning off signaling pathways, is consistent with possible tumor-suppression action. When phosphatase activity is lost because of genetic mutation, signaling pathways can become activated constitutively, resulting in aberrant proliferation. PTEN mutations have been found in as many as $30 \%$ of glioblastomas, more commonly in primary glioblastoma multiformes [22]. Apart from that, $\beta$ Catenin, P16 del, INK 4A, $\mathrm{Rb}, \mathrm{LOH} 19 \mathrm{q}, \mathrm{IDH} 1$, IDH 2, NF- $\mathrm{B}$ (nuclear factor kappa-lightchain-enhancer of activated B cell) have also been noted to play roles in various studies.

\section{Review of Literature and Discussion}

A.M. Stark et al studied 27 cases age of them are from $21 \mathrm{yrs}$ to older of histologically confirmed GBM with a history of total tumour resection at initial craniotomy. They all underwent Radiotherapy (atleast 54Gy) and 17 of them received adjuvant chemotherapy. Biopsy at recraniotomy were studied by immunohistochemistry for P53(D0-1), anti MDM2(IF-2), anti EGFR(H11) and anti MSH2(AB-1). Study revealed in comparison with initial tumour recurrent lesions were characterised by reduced expression of $\mathrm{P} 53(\mathrm{p}<0.0001)$ and $\mathrm{MSH} 2(\mathrm{p}<0.0012)$ while the numbers of $\operatorname{MDM} 2(p=0.02), \operatorname{EGFR}(p<0.0001)$ and MSH2 positive specimen were reduced $(\mathrm{p}<0.0001)$ [23].

K.C. Jain et al. [24] studied 10 patients for the role of loss of heterozygosity of P53 gene in paired primary and recurrent glioma and tried to correlate it with degree of malignancy and recurrence interval. In the study no indicative correlation was found between P53 heterozygosity status on one hand and grade of malignancy and recurrence on the other [24].

Gomori et al. [25] studied 7 patients of primary glioma and recurrent glioma (developed later) for microsatellite instability (MSI) for causation of recurrence. They found no significance of hMLH1 and hMSH2 gene in the causation of recurrence, rather these MSI were associated with primary glioma but not with recurrent one. They suggested about the clonal selectivity regarding the origin of recurrent gliomas [25].

E A Maher et al. [26] studied 20 primary and (17) secondary glioblastoma cases for evaluation of their histogenesis at molecular level. Study revealed EGFR exclusive to primary glioblastoma case whereas P53 alteration was associated with recurrent and low-grade astrocytoma cases [26].

Haradak et al. [27] studied 42 glioblastoma biopsy sample for evaluation of role of P53 and Telomerase activity in their causation. They found significantly high level of telomerase activity in secondary glioblastoma cases [27].

A Das et al. [28] studied glioblastoma cases in Asian population and found overexpression of EGFR and MDM2 in primary cases and overexpression of P53(96\% of the sample) and mutation of P53(18\%) in 7 out of 39 cases of overexpressed P53 cases and overexpression of PDGFR (significant) [28].

A Nayek et al. [29] studied 152 patients with astrocytoma and 28 with oligodendroglioma for overexpresion of P53.Study revealed overall $52 \%$ of supratentorial astrocytic tumour show immunoreactivity with no correlation to histological grade. $58.8 \%$ of diffuse astrocytoma (grade II), 53.8\% anaplastic variety (grade III) and $50 \%$ of glioblastoma (grade IV) were positive for P53. In contrast all infratentorial tumour were negative for P53 except 1 brainstem gloma. Similarly, pilocytic astrocytoma were uniformly negative forP53 irrespective of location [29].

M Puputti et al. [30] studied in astrocytoma, anapalastic astrocytoma, glioblastoma cases for KIT, PDGFRA, VEGFR2 and EGFR amplification and overexpression revealed that KIT and PDGFRA were more frequent in anaplastic variant than astrocytoma (grade II), oligodendroglioma (28\% versus $5 \%$, $\mathrm{p}=0.012$ ) and ( $33 \%$ versus $2 \%, \mathrm{p}=0.0008)$ respectively. VEGFR2 amplification noted in $6-17 \%$ of glioma cases at diagnosis and EGFR amplification in $0-12 \%$. Amplified KIT and PDGFRA was more often present at glioma recurrence than at the time of first diagnosis $(p=0.0066)$ and $(p=0.061)$ respectively, whereas VEGFR2 and EGFR amplification did not $(p=0.83)$ and $(p=0.51)$ respectively [30].

D Kita et al. [31] analysed 107 primary (de novo) and 32 secondary Glioblastoma cases through SSCP followed by DNA sequencing. They found amplification of EGFR in 33(31\%) in primary and 4 (13\%) in secondary GBM [31].

Viana-Pereira et al. [32] studied EGFR overexpression, EGFRvIII mutation and EGFR amplification, determined by immunohistochemistry and chromogenic in situ hybridization ( $\mathrm{CISH}$ ) in 27 primary glioblastoma, 24 anaplastic oligodendrogliomas (AO) and four anaplastic oligoastrocytomas (AOA) and found EGFR overexpression was associated with EGFR amplification, being found in $48 \%$ and 53\% GBM, 33\% and $40 \%$ $\mathrm{AO}$ and $75 \%$ and $67 \% \mathrm{AOA}$, respectively. EGFRvIII was found in 
$22 \%$ GBM. $8 \%$ AO and was absent in AOA. No association was observed between EGFR alterations and patient survival. Thus, for the first time, they observed EGFR molecular alterations in Portuguese patients with malignant glioma and identified a subpopulation of patients presenting putative biomarkers for EGFR-based therapies [32].

E.W. Newcomb et al. [33] investigated Using molecular genetic analysis for p53 gene mutations together with immunophenotyping for overexpression of EGFR, up to four GBM variants can be distinguished, including the p53+/EGFRprogressive or the p53-/EGFR+ de novo variant. They examined the survival of 80 adult patients diagnosed with astrocytic Glioblastoma stratified by age category ( $>40,41-60$ or $61-80)$ to determine whether alterations in any one given growth control gene or whether different genetic variants of Glioblastoma (progressive versus de novo) were associated with different survival outcomes. Survival testing using Kaplan-Meier plots for Glioblastoma patients with or without altered expression of $\mathrm{p} 16$, p53, EGFR, MDM2 or Bcl-2 showed no significant differences by age group or by gene expression indicating a lack of prognostic value for Glioblastoma. Also the clinical outcome among patients with Glioblastoma showed no significant differences within each age category for any Glioblastoma variant including the progressive and de novo Glioblastoma variants indicating similar biologic behavior despite different genotypes. Using a pairwise comparison, one-third of the Glioblastoma with normal p16 expression showed accumulation of MDM2 protein and this association approached statistical significance $(0.01<\mathrm{P}<$ 0.05) using the Bonferroni procedure. These Glioblastoma may represent a variant in which the p19ARF/MDM2/p53 pathway may be deregulated rather than the $\mathrm{p} 16 /$ cyclin $\mathrm{D}-\mathrm{CDK} 4 / \mathrm{Rb}$ pathway [33].

J Hu et al. [34] investigated molecular genetic alterations associated with primary and corresponding recurrent glioblastoma cases and to identify which chromosomal regions of the whole genome may be involved in the recurrence of primary Glioblastoma. A high-resolution allelotyping study of one patient's primary GBM and corresponding recurrent GBM was performed by PCR-based loss of heterozygosity (LOH) analysis with the use of 382 fluorescent dye-labeled polymorphic microsatellite markers covering all 22 autosomes. Study revealed chromosome $9 p$ and $10 q$ may be involved in the development of this Glioblastoma. Although histopathological diagnoses of the primary and corresponding recurrent tumor are identical, the recurrence of Glioblastoma is characterized by an increased involvement of molecular genetic abnormalities and may be accompanied by inactivation of more tumor suppressor gene suggesting further study [34]

Smith et al. [35] analysed 174 (64 AAs and 111 GBM) high grade glioma patients by FISH technique and found EGFR amplification in 11 out of $63(17 \%)$ in AAs and 46 out of 111 (41\%) in GBMs. PTEN mutation in11/62 (15\%) of AAs \&
37/110 (34\%) of GBMs. Thus both the above markers showed significantly increased value with the higher grade of tumours [35].

Fuller et al. [36] observed 508 malignant glioma cases (WHO grade III \& IV) after performing TMA-FISH and found EGFR amplification in $37 \%$ of GBMs but in $0 \%$ of AAs. PTEN mutation was found in $42 \%$ of GBMs \& $4 \%$ in AAs. Thus expression of both the above markers showed to be associated with high grade tumours [36].

A B Heimberger et al. [37] studied 54 patients of GBM biopsed or partially/subtotally resected and underwent adjuvant conformal radiation and chemotherapy. Their EGFR and EGFRvIII status was determined by immunohistochemistry and KaplanMeier estimates of overall survival were obtained. It showed $42.6 \%(n=23)$ of patients failed to express EGFR, 25.9\% ( $n=14)$ had over expression of the wild-type EGFR only and $31.5 \%$ ( $\mathrm{n}=$ 17) expressed the EGFRvIII. Patients within groups expressing the EGFR, EGFRvIII, or lacking EGFR expression did not differ in age, Karnofsky Performance Scale (KPS) score, extent of tumor resection. They all had received postoperative radiation and chemotherapy. The median overall survival times for patients with tumors having no EGFR expression, over expressed EGFR only, or EGFRvIII were 12.3 (95\% CI, 8.04-16.56), 11.03 (95\% CI, 10.18-11.89) and 14.07 (95\% CI, 7.39-20.74) months, respectively, log rank test $\mathrm{p}>0.05)$. Patients with tumors that over expressed the EGFR and EGFRvIII were more likely to present with ependymal spread, $21.4 \%$ and $35.3 \%$ respectively, compared to those patients whose GBM failed to express either marker, $13.0 \%$, although the difference was not statistically significant. There was no significant difference in multifocal disease or gliomatosis cerebri among EGFR expression groups [37].

A M Stark et al. [38] (year 2010) studied patients of glioblastoma to compare four performance score, [Karnofsky Performance Score (KPS), Glasgow Outcome Score (GOS), Modified ranking score, and Medical Research Council brain prognostic index (MRC)] with patient survival and to compare the prognostic value of performance with that of other prognostic factors. Univariate and multivariate survival analysis was used. Survival analysis revealed a high correlation to survival for all four scores. The maximum derivation of the curves was shown for the MRC and GOS. Performance had more clinical impact in determining patient survival than age and tumour resection [38].

A M Stark et al. [39] studied 143 patients (77 male, 66 female) of glioblastoma, for assessing a possible correlation between the immunohistochemical p53, Mdm2, EGFR and Msh2 expression with age. Immunohistochemical staining (IHC) was performed using anti-p53 (clone D0-1), anti-Mdm2 (clone IF-2), anti-EGFR (clone H11) and anti-Msh2 antibodies (clone AB-1). The results were compared with the Ki67/MIB-1 proliferation index (Ki67 PI) and patient survival. It was found that p53 


\section{Juniper Online Journal of Case Studies}

protein expression was significantly decreasing with advanced age $(\mathrm{p}<0.05)$ whereas EGFR and Mdm2 expression was increasing ( $p<0.05 ; p=0.01)$. Msh2 expression was unrelated to age. Multivariate analysis revealed Msh2 protein expression as a significant predictor of prolonged survival $(p=0.004)$ whereas p53, Mdm2 and EGFR were not associated with patient survival [39].

\section{Discussion}

Over the past decade, the concept of different genetic pathways leading to the common phenotypic endpoint (i.e., GBM) has gained general acceptance. Genetically, primary and secondary glioblastomas show little overlap and constitute different disease entities. Studies are beginning to assess the prognoses associated with different mutations. So gliomagenesis is heterogenous origin be of primary or recurrent variety. At the molecular level, it has been noted that primary and recurrent variety may have differences regarding their tumorigenesis, immunohistochemistry status and prognosis. Information regarding p53 and EGFR expression in recurrence of gliopma tumorigenesis is still sparse.

\section{Acknowledgement}

Authors acknowledged contribution from all patients for giving consent for this study and its possible publication.

\section{References}

1. Louis DN, Ohgaki H, Wiestler OD, Cavenee WK, Burger PC (2007) WHO Classification of tumours of the central nervous system. Acta Neuropathologica 114(2): 97-109.

2. Duport CD, Scheithauer B, O Fallon J, Kelly P (1988) Grading of astrocytomas. A simple and reproducible method. Cancer 62(10): 2152-2165.

3. Shaw EG, Scheithauer BW, O Fallon JR, Tazelaar HD, Davis DH (1992) Oligodendrogliomas the Mayo clinic experience. J Neuroserg 76(3): 428-434.

4. Robertson PL, Zeltzer PM, Boyett JM, Rorke LB, Allen JC, et al. (1998) Survival and prognostic factors following radiation therapy and chemotherapy for ependymoma in children: a report of the Children's Cancer Group. J Neurosurg 88(4): 695-703.

5. Kudo H, Di S, Tamaki N, Nishida Y, Matsumoto S (1990) Ependymoma diagnosed in the first year of life in japan in collaboration with the International Society for Pediatric Neurosurgery. Child Nerv Syst 6(7): 375-378.

6. Pollack IF, Gerszten PC, Martinej AG, Lo KH, Schultz B, et al. (1995) Intracranial ependymomas of childhood:long term outcome and prognostic factors. Neurosurgery 37(4): 655-666.

7. Zulch KJ (1986) Brain Tumours. Their Biology and pathology. ( $3^{\text {rd }}$ edn), Springer Verlag: Berlin Heidelber, New York, USA.

8. Kleihues P, Ohgaki H (1999) Primary and secondary glioblastoma: from concept to clinical diagnosis. Neuro Oncol 1(1): 44-51.

9. Kim TS, Halliday AL, Hedley W, Convery K (1991) Correlates of survival and the Daumas-Duprt grading system for astrocytomas. J Neurosurg 74(1): 27-37.

10. Lee TT, Manzaro GR (1997) Third ventricular glioblastoma multiforme: case report. Neurosurg Review 20(4): 291-294.
11. Anzil AP (1970) Glioblastoma multiforme with extra cranial metastasis in absence of previous craniotomy. Case report. J Neurosurg 33(1): 8889.

12. Burger PC, Heinz ER, Shibata T, Kleihues P (1988) Topographic anatomy and CT correlations in the untreated glioblastoma multiforme. J Neurosurg 68(5): 698-704.

13. Patronas NJ, Di Chiro G, Kufta C, Bairamian D, Kornblith PL, et al. (1985) Prediction of survival in glioma patients by means of positron emission tomography. J Neurosurg 62(6): 816-822.

14. Lantos PL, Vandenberg SR, Kleihous P (1996) Tumours of the nervous system. In: Graham D, Lantos P L (Eds.), Greenfield's Neuropathology. ( $6^{\text {th }}$ edn), Arnold: London, UK, pp.583-879.

15. Lomax ME, Barnes DM, Gilchrist R, Picksley SM, Varley JM, et al. (1997) Two functional assays employed to detect an unusual mutation in the oligomerisation domain of p53 in a Li-Fraumeni-like family. Oncogene 14(15): 1869-1874.

16. Greenblatt MS, Bennett WP, Hollstein M, Harris CC (1999) Mutations in the p53 tumor suppressor gene: clues to cancer etiology and molecular pathogenesis. Cancer Res 54(18): 4855-4878.

17. Watanabe K, Sato K, Biernat W, Tachibana O, von Ammon K, et al. (1997) Incidence and timing of p53 mutations during astrocytoma progression in patients with multiple biopsies. Clin Cancer Res 3(4): 523-530.

18. Olayioye MA, Neve RM, Lane HA, Hynes NE (2000) The ErbB signaling network: Receptor heterodimerization in development and cancer. EMBO J 19(13): 3159-3167.

19. Jorissen RN, Walker F, Pouliot N, Garrett TP, Ward CW, et al. (2003) Epidermal growth factor receptor: Mechanisms of activation and signalling. Exp Cell Res 284(1): 31-53.

20. Pelloski CE, Ballman KV, Furth AF, Zhang L, Lin E, et al. (2007) Epidermal growth factor receptor variant III status defines clinically distinct subtypes of glioblastoma. J Clin Oncol 25(16): 2288-2294.

21. Korkolopoulou P, Christodoulou P, Kouzelis K, Hadjiyannakis M, Priftis A, et al. (1997) MDM2 and p53 expression in gliomas: a multivariate survival analysis including proliferation markers and epidermal growth factor receptor. Br J Cancer 75(9): 1269-1278.

22. Furnari FB, Fenton T, Bachoo RM, Mukasa A, Stommel JM, et al. (2007) Malignant astrocytic glioma: genetics, biology, and paths to treatment. Genes Dev 21(21): 2683-2710.

23. Stark AM, Witzel P, Strege RJ, Hugo HH, Mehdorn HM (2003) p53, mdm2, EGFR, and msh2 expression in paired initial and recurrent glioblastoma multiforme. J Neurol Neurosurg Psychiatry 74(6): 779783.

24. Jain KC, Chattopadhyay P, Sarkar C, Sinha S, Mahapatra AK (1999) A pilot study of recurrence of human glial tumours in light of p53 heterozygosity status. Journal of Biosciences 24(4): 477-481.

25. Gomori É, Fulop Z, Meszaros I, Doczi T, Matolscy A (2002) Microsatellite Analysis of Primary and Recurrent Glial Tumors Suggests Different Modalities of Clonal Evolution of Tumor Cells. Journal of Neuropathology \& Experimental Neurology 61(5): 396-402.

26. Maher EA, Brennan C, Wen PY, Durso L, Ligon KL, et al. (2006) Marked genomic differences characterize primary and secondary glioblastoma subtypes and identify two distinct molecularand clinical secondary glioblastoma entities. Cancer Res 66(23): 11502-11513.

27. Harada K, Kurisu K, Tahara H, Tahara E, Ide T, et al. (2000) Telomerase activity in primary and secondary glioblastomas multiforme as a novel molecular tumor marker. J Neurosurg 93(4): 618-625.

28. Das A, Tan WL, Teo J, Smith DR (2002) Glioblastoma multiforme in an Asian population: evidence for a distinct genetic pathway. J Neurooncol 60(2): 117-125. 
29. Anupma Nayak, Ralte AM, Sharma MC, Singh VP, Mahapatra AK, et al. (2004) p53 protein alterations in adult astrocytic tumors and Oligodendrogliomas. Neurology India 52(2): 228-232.

30. Puputti M, Tynninen O, Sihto H, Blom T, Mäenpää H, et al. (2006) Amplification of KIT, PDGFRA, VEGFR2, and EGFR in gliomas. Mol Cancer Res 4(12): 927-934.

31. Kita D, Yonekawa Y, Weller M, Ohgaki H (2007) PIK3CA alterations in primary (de novo) and secondary glioblastomas. Acta Neuropathol 113(3): 295-302.

32. Viana-Pereira M, Lopes JM, Little S, Milanezi F, Basto D, et al. (2008) Analysis of EGFR overexpression, EGFR gene amplification and the EGFRvIII mutation in Portuguese high-grade gliomas. Anticancer Res 28(2A): 913-920.

33. Newcomb EW, Cohen H, Lee SR, Bhalla SK, Bloom J, et al. (1998) Survival of patients with glioblastoma multiforme is not influenced by altered expression of p16, p53, EGFR, MDM2 or Bcl-2 genes. Brain Pathol 8(4): 655-667.

34. Hu J, Jiang CC, Ng HK, Pang JC, Tong CY, et al. (2004) A genome-wide allelotype study of primary and corresponding recurrent glioblastoma multiforme in one patient. Chin Med J (Engl) 117(3): 456-458.

35. Smith JS, Tachibana I, Passe SM, Huntley BK, Borell TJ, et al. (2001) PTEN Mutation, EGFR amplification, and outcomein patients with anaplastic astrocytoma and glioblastoma multiforme. J Natl Cancer Inst 93(16): 1246-1256.

36. Fuller CE, Wang H, Zhang W, Fuller GN, Perry A (2002) Highthroughput molecular profiling of high-grade astrocytomas: the utility of fluorescence in situ hybridization on tissue microarrays (TMAFISH). J Neuropathol Exp Neurol 61(12): 1078-1084.

37. Heimberger AB, Suki D, Yang D, Shi W, Aldape K (2005) The natural history of EGFR and EGFRvIII in glioblastoma patients. J Transl Med 3: 38 .

38. Stark AM, Stepper W, Mehdorn HM (2010) Outcome evaluation in glioblastoma patients using different ranking scores: KPS, GOS, mRS and MRC. Eur J Cancer Care (Engl) 19(1): 39-44.

39. Stark AM, Hugo HH, Witzel P, Mihajlovic Z, Mehdorn HM (2003) Kl Zentralbl Neurochir. nik für Neurochirurgie im Universitäts klinikum Kiel 64(1): 30-36.

\section{Your next submission with Juniper Publishers will reach you the below assets}

- Quality Editorial service

- Swift Peer Review

- Reprints availability

- E-prints Service

- Manuscript Podcast for convenient understanding

- Global attainment for your research

- Manuscript accessibility in different formats

( Pdf, E-pub, Full Text, Audio)

- Unceasing customer service

Track the below URL for one-step submission https://juniperpublishers.com/online-submission.php 